\title{
Monetary Policy and Stable Indeterminacy with Inertia*
}

\author{
George W. Evans \\ University of Oregon
}

\author{
Bruce McGough \\ Oregon State University
}

March 29, 2004

\begin{abstract}
We examine existence and stability under learning of sunspot equilibria in a New Keynesian model incorporating inertia. Indeterminacy remains prevalent, stable sunspots abound, and inertia in IS and AS relations do not significantly impact the policy region containing stable sunspots.
\end{abstract}

JEL classification: E52, E32, D83, D84.

Keywords: Monetary Policy, sunspots, learning, stability.

\section{Introduction}

It is well known that when closed with Taylor-type rules, New Keynesian models of monetary policy can exhibit indeterminacy and sunspot equilibria in large regions of the parameter space: see for example [1], [21] and [18]. Some authors, including [6], have even suggested that the volatile inflation and output of the seventies was in part due to sunspot phenomena.

Other authors have down-played the applied importance of sunspot equilibria, arguing that in general they seem to be unstable under learning. Although it has been shown by [20] that stable sunspots can exist in simple overlapping generations models,${ }^{1}$ the sunspots in many calibrated applied models

*This material is based upon work supported by the National Science Foundation under Grant No. 0136848.

${ }^{1}$ For the local stability conditions see [7] and [9]. 
are lacking this necessary stability. For example [8] show that sunspots in the Farmer-Guo model are unstable, and [10] describe a stability puzzle surrounding the lack of stable indeterminacies in a host of non-convex RBC-type models. In contrast, in [11] we found that New Keynesian monetary models exhibit stable sunspots provided the policy rule is forward looking, and provided agents use forecasting rules consistent with what we called the common factor representation of a given sunspot equilibrium. ${ }^{2}$

Some researchers have pointed out that the presence of inertia in a model may significantly alter the associated regions (in policy space) of indeterminacy, shrinking them relative to the determinate regions and thus rendering less compelling the concern of indeterminacy. (See, e.g. the discussion of this issue in [16]). Clearly, the relative weight of inertial and forward looking elements can in general affect both indeterminacy and stability under learning, and in New Keynesian models [3] and [11] found that increased inertia in the policy rule (PR) in some cases enhances the likelihood of stable determinacy. Since substantial levels of inertia are often present in applied models of the economy, this is an issue of considerable practical importance.

In [11], we did allow for inertia in the aggregate supply (AS) relation. However, our study did not include an inertial term in the IS curve, and the importance of inertia was not our focus. In the current paper this gap is filled by the specification of an IS relation with inertia and a thorough and symmetric study of the impact of all three types of inertia (i.e. inertia in the AS, IS, and PR equations) on the regions in policy space associated with indeterminacy and stability.

We find that even models exhibiting a high degree of inertia still have large regions of indeterminacy. Furthermore, the regions of stable indeterminacy do not appear to be altered by the inertial components of the IS and AS curves. While these structural inertial terms may eliminate some regions of unstable indeterminacy, they tend to be replaced by regions of explosiveness or unstable determinacy, and thus may still result in bad outcomes.

\footnotetext{
${ }^{2}$ For the theory of common factor representations see [12]. Stability of determinate equilibria in these types of monetary models was studied in [2] and [3]. Stability of certain classes of sunspots was previously examined in [15] and in [4]. The latter paper focuses on the impact of information sets and on the timing of cash-in-advance constraints.
} 


\section{Theory}

We use a version of the New Keynesian model incorporating inertia:

$$
\begin{aligned}
I S: x_{t} & =-\phi\left(i_{t}-E_{t} \pi_{t+1}\right)+\psi\left(\delta E_{t} x_{t+1}+(1-\delta) x_{t-1}\right)+g_{t} \\
A S: \pi_{t} & =\beta\left(\gamma E_{t} \pi_{t+1}+(1-\gamma) \pi_{t-1}\right)+\lambda x_{t}+u_{t} \\
P R: i_{t} & =\theta i_{t-1}+(1-\theta) \alpha_{x} E_{t} x_{t+1}+(1-\theta) \alpha_{\pi} E_{t} \pi_{t+1}
\end{aligned}
$$

Here $x_{t}$ is the proportional output gap, $\pi_{t}$ is the inflation rate, and $g_{t}$ and $u_{t}$ are independent, exogenous, stationary, zero mean $\operatorname{AR}(1)$ shocks with damping parameters $0 \leq \rho_{g}, \rho_{u}<1$ respectively. We assume $0<\delta, \gamma, \theta<1$.

Equation (1) is the forward looking "IS" relationship with an inertial component $x_{t-1}$ consistent with habit formation: e.g. see Ch. 4 of [22]. Equation (2) is the forward-looking New Keynesian "AS" relationship based on "Calvo pricing," and employed in [5] and Chs. 3, 6 of [22]. The specification of the AS curve with inertial term $\pi_{t-1}$ is similar in spirit to [13], Section 4 of [14], and Ch. 3, Section 3.2 and Chapter 6, Section 4.6 of [22]. The monetary policy rule (3) is a forward looking version of the Taylor rule, in which the interest rate $i_{t}$ is raised in response to forecasts of higher inflation or output and an interest rate smoothing term is included. This type of rule figures prominently in the literature, with $\alpha_{\pi}>1$ usually recommended.

A rational expectations equilibrium (REE) is any non-explosive solution to the system $(1)-(3)$. For a given specification of the structural parameters $\{\phi, \lambda, \psi, \beta, \rho\}$, inertial parameters $\{\theta, \gamma, \delta\}$, and policy parameters $\left\{\alpha_{x}, \alpha_{\pi}\right\}$, the model is said to be determinate if there is a unique REE, indeterminate if there are multiple REE, and explosive if there are no REE.

Let $y=(x, \pi, i)^{\prime}$ and $\hat{g}=(g, u)^{\prime}$. In the determinate case the unique REE can be represented in minimal state variable (MSV) form as

$$
y_{t}=b y_{t-1}+c \hat{g}_{t}
$$

If the model is indeterminate, any given equilibrium has representations of two distinct functional forms. General Form representations are

$$
y_{t}=b y_{t-1}+h y_{t-2}+c \hat{g}_{t}+d \hat{g}_{t-1}+e \xi_{t},
$$

where $\xi_{t}$ is an exogenous martingale difference sequence capturing the sunspot dependence. Common Factor representations are

$$
y_{t}=b y_{t-1}+c \hat{g}_{t}+d \zeta_{t}
$$


where $\zeta_{t}$ is an exogenous serially correlated process capturing the sunspot. A common factor representation may be thought of as an MSV representation augmented by a serially correlated sunspot. Details on constructing these types of representations are given in $[11] .{ }^{3}$ Existence of representations taking the form (4) requires a sufficient number of real roots.

To examine stability under learning of a given representation, we posit a linear forecasting model, or perceived law of motion (PLM), consistent with the representation of interest:

$$
y_{t}=\Theta X_{t}
$$

Here $X_{t}$ is the collection of relevant regressors and $\Theta$ the vector of perceived coefficients: for example, if we are studying the stability of common factor representations, $X_{t}$ would include $y_{t-1}, \hat{g}_{t}, \zeta_{t}$ and a constant. Agents are assumed to form expectations of $y_{t+1}$ using this forecasting model. Inserting these expectations into the economic model of the economy $(1)-(3)$, we obtain the implied actual law of motion (ALM) for the economy. For wellspecified PLMs, the ALM will depend upon the same regressors, though the true parameters may differ. We write the ALM as

$$
y_{t}=T(\Theta) X_{t}
$$

to emphasize the existence of a map $T$ taking perceived to actual coefficients.

A fixed point $\Theta^{*}$ of $T(\Theta)$ defines both an REE and an associated representation $y_{t}=\Theta^{*} X_{t}$. We say the REE and the associated representation are E-stable provided $\Theta^{*}$ is a locally asymptotically stable fixed point of the differential equation:

$$
\frac{d \Theta}{d \tau}=T(\Theta)-\Theta
$$

The E-stability Principle tells us that an E-stable REE will be locally stable under RLS (recursive least squares) and closely related learning algorithms. The intuition of the E-stability Principle is straight-forward. Under RLS estimates are updated by moving in a direction dictated by orthogonality of forecast errors to regressors. The above differential equation well approximates the essential component of this adjustment by directing the perceived

\footnotetext{
${ }^{3}$ Depending on the order of indeterminacy, the sunspot will be one or two dimensional. The damping parameter(s) in the autoregressive structure of $\zeta$ are pinned down by the parameters of the model and are said to satisfy the "resonance frequency" condition.
} 
parameters $\Theta$ to move in the direction of the true parameters $T(\Theta)$. Estability implies that such motion will eventually lead to the REE. There is a deep connection between E-stability and stability under least squares learning: for details as they pertain to models of the form considered here, see [11], and for general information on learning see [8].

\section{Results}

To present our results we employ the following notation: $\mathrm{SD}=$ stable determinacy; $\mathrm{UD}=$ unstable determinacy; $\mathrm{SI}=$ stable indeterminacy; $\mathrm{UI}=$ unstable indeterminacy; $\mathrm{E}=$ explosive; and finally, $\mathbb{C}$ will be used to indicate that at most one eigenvalue is real so that common factor sunspots do not exist. Thus if we say that for given parameters the model is SI, then there are multiple equilibria with a representation that is stable under learning.

Analytic intractability forces us to resort to numerical techniques. We analyze three different calibrations of the structural parameters, due to [21], [6] and [17], as follows: W: $\phi=1 / .157, \lambda=.024$; CGG: $\phi=4, \lambda=.075$; and $\mathrm{MN}: \phi=.164, \lambda=.3 .^{4}$ In all cases, we set $\psi=1$, as is standard, and $\beta=1$ (the other standard calibration, setting $\beta$ equal to the discount factor, that is, $\beta=.99$, was also considered).

For each calibration we considered every permutation of inertial parameters with $\theta, \gamma, \delta \in\{.1, .5, .9\}$ and a $20 \times 20$ point lattice imposed on the policy space $0 \leq \alpha_{\pi} \leq 5,0 \leq \alpha_{x} \leq 5$. Each lattice point thus fully specifies a model, and the associated stability and determinacy properties were recorded. In all, over 10,000 model specifications were analyzed, making the careful reporting of all results intractable; therefore, we rely on a somewhat qualitative method of presentation, with details of our results given only for the Woodford calibration.

Under the Woodford calibration, for any given specification of inertial parameters, the $5 \times 5\left(\alpha_{\pi}, \alpha_{x}\right)$ policy space can be roughly described as looking like either Figure 1 or Figure 2 below. Note that Figure 1 divides the policy space into four regions and Figure 2 divides it into three regions, and these regions are labeled counter-clockwise. (The figures display only a portion of the benchmark space.) No stable general form sunspots were found for any of the three calibrations.

\footnotetext{
${ }^{4}$ Calibrations are for quarterly data with $i_{t}$ and $\pi_{t}$ measured at quarterly rates. The CGG calibration (based on annualized rates) is therefore adjusted accordingly.
} 
For $\theta=.1$ or .5 policy space takes the form of Figure 1 . If $\theta=.1$ then $\alpha_{x}^{c} \approx .5$ and if $\theta=.5$ then $\alpha_{x}^{c} \approx 1$. Region I is always SI and region IV is always SD. Region II may be UD, UI or $\mathbb{C}$ and region III may be E, UI, or $\mathbb{C}$, depending on the values of $\delta$ and $\gamma$. Varying $\delta$ and $\gamma$ does not impact the size of the regions and only affects the properties of regions II and III. ${ }^{5}$

If $\theta=.9$ policy space takes the form of Figure 2. For $\gamma, \delta=.1$ the hypotenuse of region III is almost horizontal at $\alpha_{x}=.5$. As $\gamma$ and $\delta$ increase the slope of the hypotenuse becomes steeper until region III vanishes. Region I is always SD, region II may be E, UI, or $\mathbb{C}$, and region III is E. Changes in $\delta$ and $\gamma$ only impact the properties of region II and the size of region III.

Setting $\beta=.99$ alters these results somewhat; the northeast section of region II is replaced by region I (both figures).

Figures Here

The results for the CGG calibration are qualitatively similar to the Woodford calibration with the exception that for $\theta=.9, \gamma=.1$, and $\delta=.5, .9$ region III of Figure 2 contains an interesting mix of explosive steady-states and stable and unstable determinacy. The results for the MN calibration, for all inertial specifications, are qualitatively like Figure 2, with region III empty unless high levels of inertia are present.

In summary, even when $\alpha_{\pi}>1$, stable indeterminacy can arise even when substantial structural inertia is present. Increased interest rate smoothing often enlarges the area of stable determinacy, but in some cases can convert stable determinacy to unstable or explosive solutions.

\section{Conclusion}

We find that even models with high levels of inertia have large regions of indeterminacy, and furthermore that the regions of stable indeterminacy do not appear to be impacted by the extent of inertia in the structural equations. While such inertial terms may eliminate some regions of unstable indeterminacy, they tend to be replaced by regions of explosiveness or unstable determinacy. Since the resulting behavior of the economy in each of these cases is highly unpredictable, we conclude that relying on structural inertia to dismiss problems of indeterminacy appears ill-advised.

\footnotetext{
${ }^{5}$ If $\theta=.5, \gamma=\delta=.1$, there is a small region $\mathrm{E}$ in the southwest corner of region $\mathrm{IV}$.
} 
In contrast, increased interest rate smoothing can diminish the dangers from indeterminacy by increasing the relative size of the region of stable determinacy. However, in some cases increased smoothing generates unstable or explosive solutions.

\section{References}

[1] B.S. Bernanke and M. Woodford. Inflation forecasts and monetary policy. Journal of Money, Credit, and Banking, 24:653-684, 1997.

[2] James Bullard and Kaushik Mitra. Learning about monetary policy rules. Journal of Monetary Economics, 49:1105-1129, 2002.

[3] James Bullard and Kaushik Mitra. Determinacy, learnability and monetary policy inertia. Working paper, Federal Reserve Bank of St. Louis, 2003.

[4] Charles T. Carlstrom and Timothy S. Fuerst. Learning and the central bank. Journal of Monetary Economics, 51:327-338, 2004.

[5] Richard Clarida, Jordi Gali, and Mark Gertler. The science of monetary policy: A new keynesian perspective. Journal of Economic Literature, 37:1661-1707, 1999.

[6] Richard Clarida, Jordi Gali, and Mark Gertler. Monetary policy rules and macroeconomic stability: Evidence and some theory. Quarterly Journal of Economics, 115:147-180, 2000.

[7] George W. Evans and Seppo Honkapohja. On the local stability of sunspot equilibria under adaptive learning rules. Journal of Economic Theory, 64:142-161, 1994.

[8] George W. Evans and Seppo Honkapohja. Learning and Expectations in Macroeconomics. Princeton University Press, Princeton, New Jersey, 2001.

[9] George W. Evans and Seppo Honkapohja. Existence of adaptively stable sunspot equilibria near an indeterminate steady state. Journal of Economic Theory, 111:125-134, 2003. 
[10] George W. Evans and Bruce McGough. Indeterminacy and the stability puzzle in non-convex economies. U. Oregon Econ. WP No. 2002-14.

[11] George W. Evans and Bruce McGough. Monetary policy, indeterminacy and learning. U. Oregon Econ. WP No. 2003-34.

[12] George W. Evans and Bruce McGough. Stable sunspot solutions in models with predetermined variables. Journal of Economic Dynamics and Control, forthcoming, 2003.

[13] Jeff Fuhrer and George Moore. Inflation persistence. Quarterly Journal of Economics, 110:127-159, 1995.

[14] Jordi Galí and Mark Gertler. Inflation dynamics: A structural econometric approach. Journal of Monetary Economics, 44:195-222, 1999.

[15] Seppo Honkapohja and Kaushik Mitra. Are non-fundamental equilibria learnable in models of monetary policy? Journal of Monetary Economics, forthcoming, 2004.

[16] Andrew Levin, Volker Wieland, and John Williams. The performance of forecast-based monetary policy rules under model uncertainty. American Economic Review, 93:622-645, 2003.

[17] Bennett T. McCallum and Edward Nelson. Performance of operational policy rules in an estimated semi-classical model. In Taylor [19], pages $15-45$.

[18] Lars E. O. Svensson and Michael Woodford. Implementing optimal policy through inflation-forecast targeting. Working paper, 1999.

[19] J.B. Taylor, editor. Monetary Policy Rules. University of Chicago Press, Chicago, 1999.

[20] Michael Woodford. Learning to believe in sunspots. Econometrica, 58:277-307, 1990.

[21] Michael Woodford. Optimal monetary policy inertia. The Manchester School, Supplement, 67:1-35, 1999.

[22] Michael Woodford. Interest and Prices: Foundations of a Theory of Monetary Policy. Princeton University Press, Princeton, NJ, 2003. 


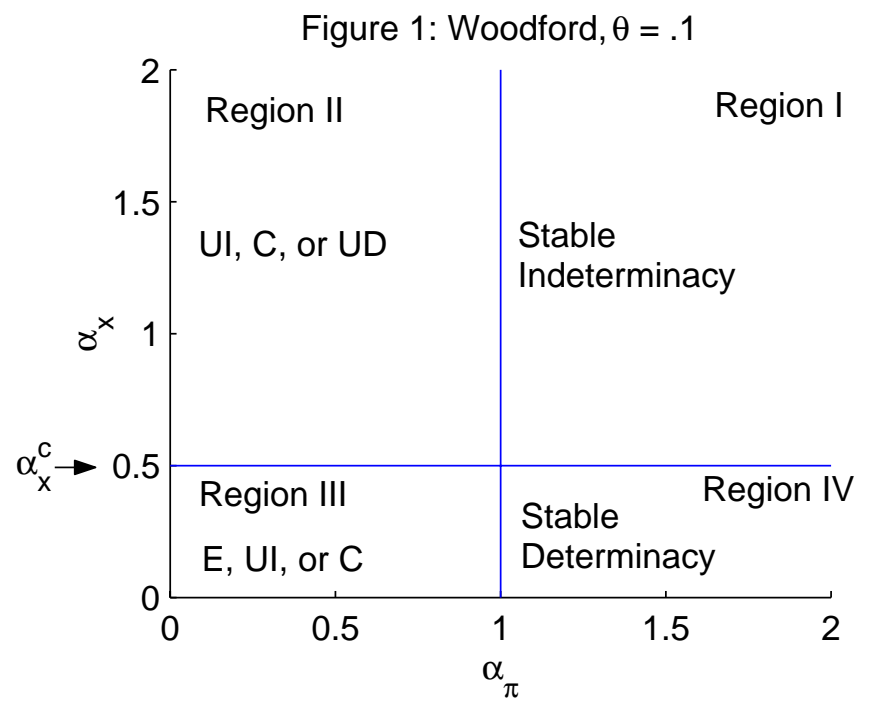

Figure 2: Woodford, $\theta=.9, \gamma=.1, \delta=.5$

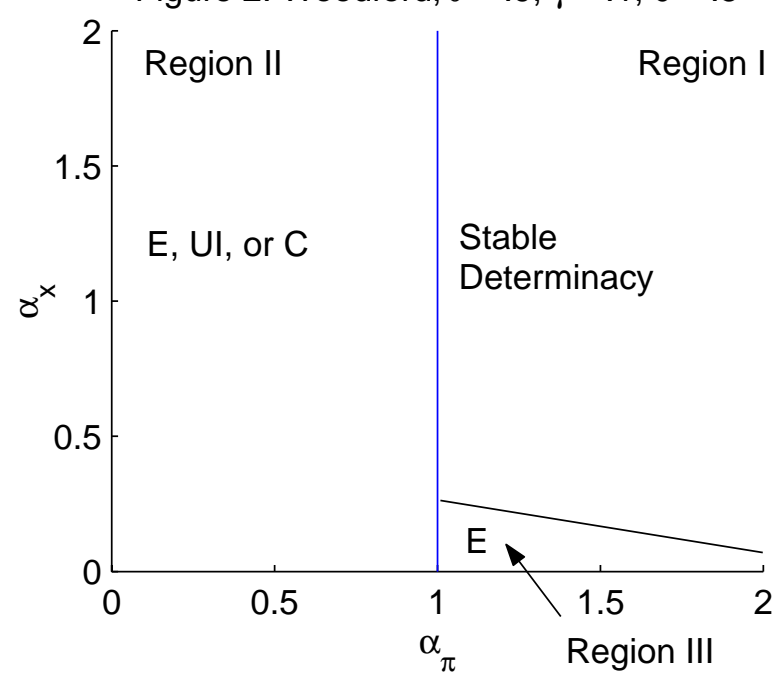

